\title{
A proof-theoretic semantics for contextual domain restriction
}

\author{
Nissim Francez \\ Computer Science Department, \\ The Technion-IIT, Haifa, Israel
}

\begin{abstract}
The paper presents a proof-theoretic semantics account of contextual domain restriction for quantified sentences in a fragment of English. First, the technique is exemplified in the more familiar firstorder logic, and in its restricted quantification variant. Then, a prooftheoretic semantics for the NL fragment is reviewed, and extended to handling contextual domain restriction. The paper addresses both the descriptive facet of the problem, deriving meaning relative to a context, as well as the fundamental aspect, defining explicitly a context (suitable for quantifier domain restriction), and specifying what it is about such a context that brings about the variation of meaning due to it.

The paper argues for the following principle (the context incorporation principle, CIP): for every quantified sentence $S$ depending on a context $c$, there exists a sentence $S^{\prime}$, the meaning of which is independent of $c$, s.t. the contextually restricted meaning of $S$ is equal to the meaning of $S^{\prime}$. Thus, the effect of a context can always be internalized. The current model-theoretic accounts of contextual domain restriction do not satisfy CIP, in that they imply intersection of some extension with an arbitrary subset of the domain, that need not be the denotation of any NL-expression.
\end{abstract}

Keywords: proof-theoretic semantics, contextual domain restriction 


\section{INTRODUGTION}

The problem of context dependence is the problem of explaining how context contributes to interpretation ...

write Stanley and Szabó (2000), who discuss a variety of special cases of the general problem of meaning variation with context. The purpose of this paper is to provide a proof-theoretic semantics $(P T S)^{1}$ (see below) for a special case of the general context dependence problem, namely quantifier domain restriction (QDR). It may well be the case that the proof-theoretic interpretation of other kinds of expressions with contextually varying meanings will require different proof-theoretic techniques than the one used here. I focus on the QDR-problem as it fits naturally into the fragment of natural language (NL) for which a PTS has been proposed before (Francez and Dyckhoff 2010; Francez et al. 2010; Francez and Ben-Avi 2014). The QDR-problem has a rich history (see Stanley and Szabó 2000, for references to earlier work), all carried out under the model-theoretic semantics (MTS) theory of meaning.

Before turning to the main semantic issue itself, I would like to recapitulate the highlights of the PTS and MTS approaches as theories of meaning. Proof-theoretic semantics is a challenging way for defining meaning, an alternative to the prevailing model-theoretic semantics, the latter equating meaning with providing truth conditions (in arbitrary models). ${ }^{2}$ The MTS approach has been criticized by several philosophers of logic and language (most notably, Dummett 1993, Prawitz 2006, Brandom 2000, Tennant 1997, and many more) as an inappropriate theory of meaning. I omit here a more detailed discussion of this criticism, often occupying full books, as justifying the approach is not the topic here. A more condensed presentation of this criticism and motivating advantages of PTS can be found in the introduction sections of (Francez and Dyckhoff 2010) and (Francez and

\footnotetext{
${ }^{1}$ A general introductory overview of PTS can be found in an entry of The Stanford Encyclopaedia of Philosophy, http://plato.stanford.edu/entries/ proof-theoretic-semantics/. Concrete references are given in the paper where appropriate.

${ }^{2}$ There is also a variant of MTS called Dynamic Semantics, which view meaning as updates of assignments. It also depends on models, entities, reference, extension, etc.
} 
Ben-Avi 2014). Initially, since the work of Gentzen (1969), PTS was conceived as a meaning-theory for logic. Recently, however, PTS has been advocated also for providing the semantics of (an extensional fragment of) NL in (Francez and Dyckhoff 2010), (Francez et al. 2010) and (Francez and Ben-Avi 2014), in contrast to the MTS approach dominant in NL formal semantics ever since Montague's seminal work. I recapitulate the essence of the PTS proposal:

- For (affirmative) sentences, replace the received approach of taking their meanings as truth conditions (in arbitrary models) by an approach taking meanings to consist of canonical derivability conditions (from suitable assumptions). This involves a dedicated proof system in natural deduction (ND) form, on which the derivability conditions are based (canonicity is explained below). In a sense, the proof system should reflect the use of the sentences in the fragment, and should allow recovering pre-theoretic properties of the meanings of these sentences such as entailment and assertability conditions. The essentials of such an ND-system are reviewed below.

- For subsentential phrases, replace taking their denotations (in arbitrary models, extensions) as meaning, by taking their contributions to the meanings (in our explication, derivability conditions) of sentences in which they occur. This adheres to Frege's context principle (Frege 1884), made more specific by the incorporation into a type-logical grammar (TLG) (see Moortgat 1997), the assumed underlying syntactic formalism. A detailed exposition of deriving meanings of subsentential phrases can be found in (Francez et al. 2010) for natural language, and in (Francez and Ben-Avi 2011) for logic.

According to the mainstream PTS programme, meaning is determined via a meaning-conferring natural-deduction proof system. An NDsystem has two families of rules for each defined expression.

Introduction rules ( $I$-rules): These are rules specifying the way a formula (sentence) having the defined expression as its main operator, the conclusion of the rule, can be deduced from other formulas, serving as premises of the rule. Such a deduction is the most direct way to deduce the conclusion. 
Elimination rules ( $E$-rules): These are rules specifying the way a consequence can be deduced from a formula (sentence) having the defined expression as its main operator, the major premise of the rule, and from some additional minor premises. Such a conclusion is the most direct conclusion of the major premise.

Both kind of rules can discharge assumptions, usually indicated with square brackets. Derivability of $\varphi$ from a collection of assumptions (a context $\left.^{3}\right) \Gamma$ is denoted $\Gamma \vdash \varphi$. Derivation $\mathscr{D}$ of $\varphi$ from $\Gamma$ is the usual recursively defined one. I use the Gentzen-Prawitz tree-like format for presenting derivations. One of its advantages over linear representations of ND-derivations, useful in the current discussion, is the convenience of representing the composition of derivations, needed for defining reductions.

An important requirement is that the ND-system should be harmonious, in that its rules have a certain balance between introduction and elimination, in order to qualify as conferring meaning. Harmony is delineated in more detail below.

A standard reference for ND-systems for logic is (Prawitz 1965). For ND-systems for an extensional ND-fragment, see (Francez and Dyckhoff 2010).

To explain the QDR-problem itself, consider the following example sentence from (from Stanley and Szabó 2000). ${ }^{4}$

$$
\text { every bottle is empty }
$$

The literal model-theoretic meaning of (1.1), involving quantification and predication, attributes the property of emptiness to every entity in a model falling under the extension of bottle. ${ }^{5}$ This truth condition is usually expressed as the inclusion of the extension of bottle in the extension of empty, alluding to the generalized quantifiers theory. The general consent is, however, that in different circumstances, to be captured by contexts, the domain of quantification is not over the

\footnotetext{
${ }^{3}$ Not to be confused with a $D R$-context $c \in C$ affecting meaning variability, as defined below.

${ }^{4}$ All the examples of natural language expressions are depicted in the sanserif font, and are always mentioned, not used.

${ }^{5}$ As noted by Glanzberg (2006), it suffices to conduct this study in an extensional fragment of NL, as intentionality seems orthogonal to QDR-problem.
} 
whole extension of bottle (all bottles in the universe); rather, it is over a restriction of this extension to one determined by a context, e.g., every bottle in a room where some party takes place in one context, or bottles in some chemistry laboratory in another context. Similarly,

$$
\text { some bottle is empty }
$$

is taken also to have contextually varying meaning, asserting that some bottle, determined by a given context, is empty, not that some bottle in the universe is empty.

A more radical approach, called contextualism, claims that there is no quantification which is not contextually restricted! Even apparently unrestricted quantification as expressed by everything or something are contextually restricted (see, for example, Glanzberg 2006).

Note that MTS in general adheres to a compositional sentential meaning assembly. The primary carriers of meaning are words, interpreted as having denotations in models (that can be rather complex), and semantic composition generates meanings for phrases until the meaning of a whole (affirmative) sentence is determined. According to this methodology, (some of) the word denotations are contextdependent, a dependence propagated to larger phrases as the interpretation process advances. I'll return to this issue in the sequel.

The general semantic problem faced in an attempt to model the variance of literal meaning with context has, according to Stanley and Szabó (2000), two facets.

Descriptive: Deriving the interpretation of some phrase relative to a context, given prior characterization of which features of a context have a bearing on the meaning of that phrase.

Fundamental: Specifying the above mentioned characterization, namely what it is about a context in virtue of which the derivation of the interpretation yields the correct meaning in that context. This specification involves some explicit definition of a context.

Thus, for (1.1), the descriptive meaning is the proper derivation of the restricted domain of quantification given a context, while the fundamental issue is what in the structure of a context determines the appropriate domain restriction.

In general, MTS has many difficulties in adequately solving the foundational aspect of contextual variance of truth conditions. A major 
contribution of the current paper is the provision of a solution, within the PTS programme, of the foundational problem.

In MTS, it is far from clear where to locate contexts with respect to a model. Stanley and Szabó (2000, p. 222), for example, admit that they avoid giving a formal characterization of the notion of a context. They just stipulate (for the QDR-problem) a certain marking in the syntactic tree (the logical form) that interfaces in a certain way with a context, and provide a description of the way this marking participates in meaning derivations (by intersecting the extension of the head noun with a set "pointed to" by the above mentioned marking). More specifically, Stanley and Szabó (2000) posit as the lexical entry of a noun, say man, (in the appropriate leaf of a syntactic tree) the following compound expression.

$$
\langle\operatorname{man}, f(i)\rangle
$$

where man is the usual extension of man (in a model), $i$ is an anchor for an object to be provided by a context, and $f$ is an anchor to a function from objects to objects, also to be provided by context. The rule for computing the extension of man in a given context $c$ is the following (in a slightly modified notation).

$$
\llbracket\langle\operatorname{man}, f(i)\rangle \rrbracket_{c} \stackrel{\text { df. }}{=} \llbracket \operatorname{man} \rrbracket \cap\{x \mid x \in c \llbracket f \rrbracket(c \llbracket i \rrbracket)\}
$$

For an argument for a different location (in the syntactic tree) of that marking (and for a rebuttal of the rejection of this location by Stanley and Szabó 2000), see (Pelletier 2003). There are also views locating this marker on the determiner node, (e.g., Westerståhl 1985). Note that in all those approaches, there is no constraint at all imposed on the set $\{x \mid x \in c \llbracket f \rrbracket(c \llbracket i \rrbracket)\}$. In particular, as is traditional in generalized quantifier theory, this set need not be the extension (in the model at hand) of any NL phrase.

I would like to claim that this degree of freedom regarding the contextual restriction set is a drawback of all the above approaches to QDR. In general, a context can be seen either as external to the interpreted sentence (e,g., a context of utterance), or explicitly contributed by some phrase in the sentence itself. For example, (1.1) can be seen as uttered in the context of bottles on some table; however, the location of the bottles can be explicitly given in the sentence itself, say by 
means of a preposition phrase, as in

$$
\text { every bottle on the table is empty }
$$

Furthermore, if the intended context is such that the salient bottles are bottles of whisky, then this again can be given by an additional explicit modification of the noun, as in

$$
\text { every whisky bottle on the table is empty }
$$

I would like to posit the following context incorporation principle as a characterization of contextually varying meaning (as far as QDR is concerned). I see this principle as originating from the semantic concept of 'meaning' (as far as it relates to contextual meaning variation), and not from any empirical fact about this variation. One certainly can conceive of contexts not having any linguistic expression. As I see it, while such contexts can contribute to other dimensions of language use, alluding to them is not part of meaning.

The context incorporation principle (CIP): For every quantified sentence $S$ with a meaning depending on a context $c$, there exists a (not necessarily unique) sentence $S^{\prime}$, s.t.

$$
\llbracket S \rrbracket_{c}=\llbracket S^{\prime} \rrbracket
$$

In other words, the effect of a given external context $c$ in terms of QDR in $S$ is always expressible by $S^{\prime}$, the meaning of which is independent of $c$ (all in the same language, or fragment thereof). Clearly, (CIP), while being allowed by (1.4), is not enforced by (1.4).

It is important to realise what is not the semantic problem discussed here, namely the determination of which is the right context for any given token of a contextually dependent meaning of a sentence. The latter issue is always determined by extra-linguistic means, independently of whether MTS or PTS are employed as the theory of meaning. Rather, the issue is how to handle contextual meaning variation once a context has been determined. Thus, if the intended context for the above example is bottles of whisky, then an explicit assumption to this effect has to be added to the given context. Once the whole intended context has been incorporated, the resulting sentence should be read as context independent. 
Finally, the consequences that can be drawn from the contextually varying meaning of an (affirmative) sentence, namely (affirmative) sentences entailed by a sentence with contextually varying meaning, which themselves have meanings varying with context, are hardly ever considered in MTS-based discussions. I will relate to them in the proposed PTS via $E$-rules in the meaning-conferring ND-system.

Note that I adopt here the view expressed in (Stanley and Szabó 2000) that contextual variance of meaning is a semantic phenomenon, and not a syntactic (ellipsis) or pragmatic (agent related) one. I would like to stress that I am investigating what (affirmative) sentences mean, and how this meaning varies with context, and not with what an agent means by asserting a sentence in a given context; the latter, involving intentions, plans etc., I do see as pragmatic. Thus, I exclude from consideration examples such as the following (from Stanley and Szabó 2000)

$$
\text { Fred is a good friend }
$$

uttered by a speaker in some circumstances to express that Fred is, actually, a terrible friend. I do not take this interpretation of (1.8) as a meaning of (1.8) in any sense of 'meaning' that semantics is concerned with.

Why adhere to CIP?

- One can see the semantic view of the QDR-phenomenon alluded above as a (partial) justification of CIP, that relates to linguistically expressible contexts. In a performative, agent related, use of a sentence with a contextually varying meaning, it is conceivable that other kinds of contextual information, not language oriented, may have an effect. For example, complex visual information in a common ground of speaker and hearer. This is certainly true for contextual resolution of deictic elements in a sentence. This would pertain to context dependence of meaning that fits a more traditional view of it, as pragmatic, not semantic.

- While I am concerned here with meanings of single (affirmative) sentences, there is clearly much semantic interest in dialogs, or discourses, which are multi-sentential linguistic entities. Adhering to CIP is compatible with identifying context with the contents of sentences previously asserted by other participants in a dialog, or preceding sentences in a discourse. From my proof-theoretic point 
of view, the PTS for such multi-sentential linguistic constructs is, at best, in its infancy. Principles like CIP may encourage further proof-theoretic investigations of such constructs.

The paper contains also a certain proof-theoretic innovation in the concept of a parametric family of introduction rules (in a naturaldeduction system), which is not directly connected to the NL set-up aimed at in the paper.

In (Francez and Wieckowski 2014), a similar approach to contextual meaning variation is applied to contextual definiteness, as in

$$
\text { the bottle is empty }
$$

where the usual existence and uniqueness, traditionally associated with definiteness, is restricted to given contexts.

In the rest of this paper, I provide a PTS for the QDR-problem, relating both to its fundamental facet as well as to its descriptive facet, by providing meaning-conferring ND-systems. I start in Section 2 with casting the solution in a logic setting, its familiarity facilitating a clearer explication of the proof-theoretic technique involved. Then, I consider a PTS for the incorporation of the QDR-problem in an extensional fragment of English, for which a PTS is provided in (Francez and Dyckhoff 2010). The paper ends with some conclusions.

2

LOGIG WITH

\section{GONTEXTUAL DOMAIN RESTRICTION}

In this section, I present a version of first-order logic (FOL) in which quantifiers are interpreted in a contextually dependent way. While there is not much interest in such a logic per se, it serves as a vehicle for a clear presentation of the ideas underlying the application of the approach to natural language. It also provides a natural host for the novel proof-theoretic concept of a parameterized family of I-rules in the intended natural-deduction meaning-conferring proof system.

First-order logic with contextual domain restriction

I assume the usual object language for FOL, with the usual definition of free/bound variables. For simplicity, a language without constant or function symbols is considered. 
Definition 2.1 (DR-context) A DR-context (domain restricting context) $c$ is a finite collection $\Gamma_{c}$ of open formulas with one free variable only; $\Gamma_{c, x}$ is the sub-collection of $\Gamma_{c}$ with $x$ as its free variable. Let $C$ be the collection of all DR-contexts.

This definition of a DR-context is certainly not the most general one for a context affecting sentential meanings, but it is intended to capture contexts as providing restriction on quantifiers, for which purpose this definition suffices. Let $\wedge_{\Gamma_{c}, x}$ be the conjunction of all open formulas in $\Gamma_{c, x}$ (that have $x$ free). I use $\Gamma_{c, x}$ and $\wedge_{\Gamma_{c, x}}$ interchangeably. I use $\Gamma_{c, x}(y)$ or $\wedge_{\Gamma_{c, x}}(y)$ to indicate the application of the condition on $x$ to another variable, $y$, resulting in a substitution of $y$ for free occurrences of $x$.

The main idea, to be captured by the rules below, is that a DRcontext provides an assumption, dischargeable in the case of universal quantification, restricting the free variable in the premise of the $I$-rule of a quantifier. Furthermore, this discharge keeps its contents excorporated from the formula (recording the context $c$ generating it in ' $F_{c}$ ').

First, recall the standard $I / E$ rules for the universal and existential quantifiers in an ND-system for FOL. ${ }^{6}$

$$
\begin{gathered}
\frac{\Gamma \vdash \varphi(x)}{\Gamma \vdash \forall x . \varphi(x)}(\forall I), x \notin \operatorname{free}(\Gamma) \quad \frac{\Gamma \vdash \forall x . \varphi(x)}{\Gamma \vdash \varphi(y)}(\forall E) \\
\frac{\Gamma \vdash \varphi(y)}{\Gamma \vdash \exists x . \varphi(x)}(\exists I) \\
\frac{\Gamma \vdash \exists x . \varphi(x) \quad \Gamma,[\varphi(y)]_{i} \vdash \chi}{\Gamma \vdash \chi}\left(\exists E^{i}\right), y \notin \operatorname{free}(\Gamma, \chi)
\end{gathered}
$$

where $\varphi(y)$ is the result of substituting $y$ for all free occurrences of $x$ in $\varphi(x)$. I now introduce a revised ND-system, in which deducibility is indicated as ' $F_{c}$ ' (in contrast to ' $\vdash$ ' indicating the deducibility in the standard system).

Restricting the universal quantifier: Recall that the intuition behind the usual $(\forall I)$-rule is that since $x$ does not occur free in $\Gamma$, it can be seen as standing for an arbitrary value, unrestricted in any way by $\Gamma$,

\footnotetext{
${ }^{6}$ I assume familiarity with standard $I / E$-rules for the propositional operators, like conjunction ' $\wedge$ ' and implication ' $\rightarrow$ '; see (Prawitz 1965) for a standard presentation.
} 
hence supporting the universal generalization embodied in the $(\forall I)$ rule. The idea behind the $I$-rule below is to restrict the generalization to those values of $x$ satisfying the contextual restriction imposed by $\Gamma_{c, x}(x)$ for a given DR-context $c$. Thereby, the same formula $\forall x . \varphi(x)$ is read differently in different DR-contexts. This is achieved by using $\Gamma_{c, x}(x)$ as a discharged assumption in the premise of the rule.

$$
\begin{gathered}
\frac{\Gamma,\left[\Gamma_{c, x}(x)\right]_{i} \vdash_{c} \varphi(x)}{\Gamma \vdash_{c} \forall x . \varphi(x)}\left(\forall I_{C}^{i}\right), x \notin \operatorname{free}(\Gamma) \\
\frac{\Gamma \vdash_{c} \forall x . \varphi(x) \quad \Gamma \vdash_{c} \wedge_{\Gamma_{c, x}}(y)}{\Gamma \vdash_{c} \varphi(y)}\left(\forall E_{C}\right)
\end{gathered}
$$

Here $\forall I_{C}$ is a family of $I$-rules indexed by DR-contexts. Every application of this rule is always by appealing to some given DR-context $c \in C$. In the interesting cases, $\Gamma_{c, x} \neq \emptyset$ will hold, though there might be vacuous DR-contexts not affecting the meaning of a universal sentence. Similarly, $\left(\forall E_{C}\right)$ is a family of $E$-rules indexed by DR-contexts. The conclusion drawn from $\forall x . \varphi(x)$ deduced relative to a DR-context $c$ is read as $\wedge_{\Gamma_{c, x}}(y) \rightarrow \varphi(y)$, namely that $y$ satisfies both $\varphi(x)$ and the contextual restriction $\wedge_{\Gamma_{c, x}}(x)$.

Restricting the existential quantifier: As for existential quantification, the contextual rules are presented below.

$$
\begin{aligned}
& \frac{\Gamma \vdash_{c} \varphi(y) \Gamma \vdash_{c} \wedge_{\Gamma_{c, x}}(y)}{\Gamma \vdash_{c} \exists x . \varphi(x)}\left(\exists I_{C}\right) \\
& \frac{\Gamma \vdash_{c} \exists x . \varphi(x) \quad \Gamma,[\varphi(y)]_{i},\left[\wedge_{\Gamma_{c, x}}(y)\right]_{j} \vdash_{c} \chi}{\Gamma \vdash_{c} \chi}\left(\exists E^{i, j}\right), y \notin \operatorname{free}(\Gamma, \chi)
\end{aligned}
$$

The $I$-rule requires that for some $y$ that satisfies the restrictions imposed by $\Gamma_{c, x}, \varphi(y)$ is derived, in order to deduce that the contextually restricted (by c) existential conclusion be derived. Recall that, like in the standard $(\exists I)$-rule, $y$ may, (and in general, will) appear free in $\Gamma$. So, the rule forces $y$ to also fall under the restriction imposed by $c$. The $E$-rule, like the standard $(\exists E)$-rule, allows the derivation of an arbitrary conclusion $\chi$, under the assumption that $\varphi$ and the contextual restriction themselves derive $\chi$ (for a fresh $y$ ). 
Remark: From the above rules, it is evident that (CIP) holds for FOL with QDR. This is true since $\Gamma_{c, x}$ (and consequently, $\wedge_{\Gamma_{c, x}}$ ) consist only of formulas in the language.

\section{Theorem 2.1 (context incorporation)}

1. $\Gamma \vdash_{c} \forall x . \varphi(x)$ iff $\Gamma \vdash \forall x . \wedge_{\Gamma_{c, x}}(x) \rightarrow \varphi(x)$.

2. $\Gamma \vdash_{c} \exists x . \varphi(x)$ iff $\Gamma \vdash \exists x . \wedge_{\Gamma_{c, x}}(x) \wedge \varphi(x)$.

\section{Proof:}

1. (a) Assume $\Gamma \vdash_{c} \forall x . \varphi(x)$ is derived by means of $\left(\forall I_{C}\right)$. By an inductive argument, the premise of $\left(\forall I_{C}\right)$, namely $\Gamma,\left[\Gamma_{c, x}(x)\right]_{i} \vdash_{c}$ $\varphi(x)$ (with $x \notin$ free $(\Gamma)$ ), implies that $\Gamma,\left[\Gamma_{c, x}(x)\right]_{i} \vdash \varphi(x)$. Therefore, by using $\left(\rightarrow I_{i}\right), \Gamma \vdash \wedge_{\Gamma_{c, x}(x)} \rightarrow \varphi(x)$, and by applying $(\forall I)$ (since $x \notin$ free $(\Gamma)$ ), we get $\Gamma \vdash \forall x . \wedge_{\Gamma_{c, x}(x)} \rightarrow \varphi(x)$.

(b) Conversely, suppose $\Gamma \vdash \forall x . \wedge_{\Gamma_{c, x}(x)} \rightarrow \varphi(x)$ is derived via $(\forall I)$ with the premise $\Gamma \vdash \wedge_{\Gamma_{c, x}(x)} \rightarrow \varphi(x)$, where $x \notin$ free $(\Gamma)$. Thus, also $\Gamma,\left[\Gamma_{c, x}(x)\right]_{i} \vdash \varphi(x)$ (due to $(\rightarrow I)$ ). By an application of $\left(\forall I_{C}^{i}\right)$ the result follows.

2. The argument for existential quantification is similar and omitted.

Here are some examples for the more interesting direction.

1. In the DR-context $c, \forall x . \varphi(x)$ is read as $\forall x . \wedge_{\Gamma_{c, x}}(x) \rightarrow \varphi(x)$. When $\varphi(x)$ is itself an implication, say $\alpha(x) \rightarrow \beta(x)$, then the result is equivalent to conjoining the antecedent with the contextual restriction, $\forall x . \alpha(x) \wedge \wedge_{\Gamma_{c, x}}(x) \rightarrow \beta(x)$.

2. Similarly, in the DR-context $c, \exists x . \varphi(x)$ is read as $\exists x . \wedge_{\Gamma_{c, x}}(x) \wedge \varphi(x)$.

Example 2.1 Suppose (1.1) is regimented by the FOL-formula $\forall x . B(x)$ $\rightarrow E(x)$ (with $B(x)$ interpreted as $x$ is a bottle and $E(x)$ as $x$ is empty). Let $c_{\text {room }}$ be a DR-context of some room, with $\Gamma_{c_{\text {room }}, x}=\{R(x)\}$ (with $R(x)$ interpreted as $x$ is in the room). Then,

$$
\frac{\Gamma,[R(x)]_{i} \vdash_{c_{\text {room }}} B(x) \rightarrow E(x)}{\Gamma \vdash_{c_{\text {room }}} \forall x . B(x) \rightarrow E(x)}\left(\forall I_{C}^{i}\right), x \notin \text { free }(\Gamma)
$$

allows the derivation of a reading of (1.1) as $\forall x . R(x) \rightarrow(B(x) \rightarrow E(x))$, equivalent to $\forall x . B(x) \wedge R(x) \rightarrow E(x)$; that is, every bottle in the room is empty. This can be seen as incorporating the DR-context into the sentence. Note that the contextually derived universally quantified sentence does not carry its contextual meaning "on its nose". To obtain 
the required reading, one has to know the DR-context in which the sentence was derived ( $c_{\text {room }}$ in this example), and consult $\Gamma_{c_{\text {room }}, x}$ to obtain this reading. Similarly,

$$
\frac{\Gamma \vdash_{c_{\text {room }}} \forall x \cdot B(x) \rightarrow E(x) \quad \Gamma \vdash_{c_{\text {room }}} R(y)}{\Gamma \vdash_{c_{\text {room }}} B(y) \rightarrow E(y)}\left(\forall E_{C}\right)
$$

allows drawing from (1.1) derived in the DR-context $c_{\text {room }}$ the conclusion $R(y) \rightarrow(B(y) \rightarrow E(y))$, equivalent to $B(y) \wedge R(y) \rightarrow E(y)$; namely a reading corresponding to if $y$ is a bottle in the room then $y$ is empty, a correct reading of the conclusion in the context $c_{\text {room }}$.

Example 2.2 Following is another example, establishing

$$
\begin{aligned}
\forall x . W(x) \wedge I(x) \rightarrow S(x), \forall y . W(y) \wedge S(y) & \rightarrow B(y) \\
& \vdash_{c} \forall z \cdot W(z) \rightarrow B(z)
\end{aligned}
$$

in a DR-context $c$ with $\Gamma_{c, z}=I(z)$. I'll return to this example below.

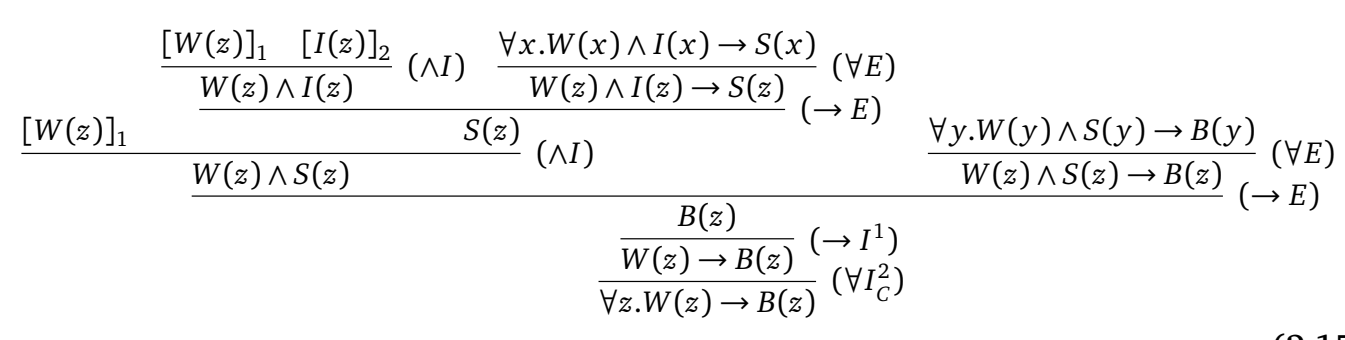

Example 2.3 The next example is of two independent QDRs by a context. It shows why the premises of the $I_{C}$-rules themselves have to use ' $\vdash_{c}$ ', and not just ' $\vdash$ '.

$$
\begin{aligned}
& \forall x \forall y \cdot M(x) \wedge Y(x) \wedge W(y) \wedge S(y) \rightarrow L(x, y), \\
& \begin{array}{l}
\forall z \cdot W(z) \wedge I(z) \rightarrow S(z) \quad{ }_{c} \quad \forall x \forall y \cdot M(x) \wedge W(y) \rightarrow L(x, y)
\end{array}
\end{aligned}
$$

where $\Gamma_{c, x}=Y(x), \Gamma_{c, y}=I(y)$. Let $\mathbf{I}$, II abbreviate, respectively, the two premises. I treat ' $\wedge$ ' as having arbitrary arity. 


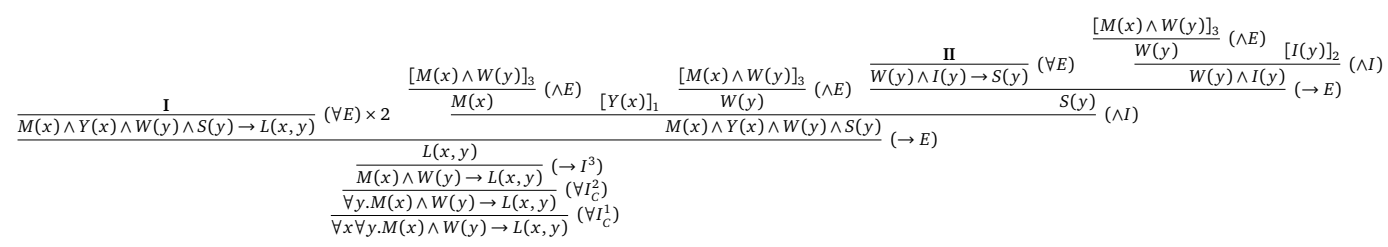

The following proposition expresses a property of the QDR-rules that will be useful below. It says that it does not matter which variable is used to express the contextual restriction, as long as it is amenable to universal generalization.

Proposition 2.1 If $\Gamma,\left[\Gamma_{c, x}(x)\right]_{i} \vdash_{c} \varphi(x)$ and $y \notin$ free $(\Gamma)$, then also $\Gamma,\left[\Gamma_{c, x}(y)\right]_{i} \vdash_{c} \varphi(y)$.

Next, consider the definition of the (reified) contextually varying sentential meanings, following the ideas in (Francez 2014c).

Definition 2.2 (canonical derivation) A derivation $\mathscr{D}$ for $\Gamma \vdash \psi$ is canonical iff it satisfies one of the following two conditions.

- The last rule applied in $\mathscr{D}$ is an $I$-rule (for the main operator of $\psi$ ).

- The last rule applied in $\mathscr{D}$ is an assumption-discharging E-rule, the major premise of which is some $\varphi$ in $\Gamma$, and its encompassed sub-derivations $\mathscr{D}_{1}, \cdots, \mathscr{D}_{n}$ are all canonical derivations of $\psi$.

Canonical derivations constitute the most direct derivations of their conclusion (though not necessarily always the shortest), and are viewed by PTS to underlie and determine meaning. Let $\llbracket \varphi \rrbracket_{\Gamma}^{c}$ denote the (possibly empty) collection of all canonical derivations of $\varphi$ from $\Gamma .{ }^{7}$

Definition 2.3 (reified meanings) The (reified) meaning of $\varphi$ is given by

$$
\llbracket \varphi \rrbracket \stackrel{\text { df. }}{=} \lambda \Gamma \cdot \llbracket \varphi \rrbracket_{\Gamma}^{c}
$$

To realize the role of canonicity in the definition of reified prooftheoretic meanings, consider the following example derivation in

\footnotetext{
${ }^{7}$ The superscript ' $c$ ' here relates to canonicity, and should not be confused with a DR-context, the latter indicated by a subscript $c$.
} 
propositional logic.

$$
\frac{\alpha \quad(\alpha \rightarrow(\varphi \wedge \psi))}{\varphi \wedge \psi}(\rightarrow E)
$$

This is a derivation of a conjunction - but not a canonical one, as it does not end with an application of $(\wedge I)$. Thus, the conjunction here was not derived according to its meaning! As far as this derivation is concerned, it could mean anything, for example, disjunction. On the other hand, the following example derivation, being canonical, is according to the conjunction's meaning.

$$
\frac{\alpha \quad \alpha \rightarrow \varphi}{\frac{\varphi}{\varphi \wedge}(\rightarrow E) \frac{\beta \quad \beta \rightarrow \psi}{\psi}(\wedge I)}(\rightarrow E)
$$

Similar examples can be found in natural language.

We can now see the difference between ordinary meanings and their contextually varying counterpart. For the context-independent meaning of $\forall x . \varphi(x)$, all the canonical derivations end with an application of the same ( $\forall I)$-rule, while for the meaning of $\forall x . \varphi(x)$ in a DR-context $c$, all canonical derivations end with an application of $\left(\forall I_{C}\right)$, varying with $c$.

As was already observed in (Francez 2014a), this reified meaning is very fine-grained, ${ }^{8}$ and a certain relaxation of it is found useful. Note that the CIP requires (strict) sameness of meaning between a contextually restricted quantified sentence and its context incorporated counterpart. However, while the relationship of canonical derivations of both are very similar - the former ending with application of $\left(\forall I_{C}\right)$ (in the universal case) whenever the latter ends with $(\rightarrow I)$ immediately followed by $(\forall I)$, they are strictly not the same! We can obtain a natural coarsening fitting also the current needs (for the CIP), still fine enough as to not identify the meanings of logically equivalent sentences as done in MTS, by introducing grounds (for assertion) for sentences (see Francez and Dyckhoff 2010 and Francez 2014c for a discussion of the role of those grounds in the PTS programme).

\footnotetext{
${ }^{8}$ For example, it is shown in (Francez 2014a) that $\llbracket \varphi \wedge(\psi \wedge \chi) \rrbracket \neq \llbracket(\varphi \wedge \psi) \wedge$ $\chi \rrbracket$.
} 
Definition 2.4 (grounds for assertion) The grounds for assertion of $\varphi$, denoted by $G \llbracket \varphi \rrbracket$, are given by

$$
G \llbracket \varphi \rrbracket \stackrel{\text { df. }}{=}\left\{\Gamma \mid \Gamma \vdash^{c} \varphi\right\}
$$

In other words, any $\Gamma$ from which there is a canonical derivation of $\varphi$ serves as a ground for asserting $\varphi$.

I now introduce an equivalence relation on meaning based on sameness of grounds (for assertion), that captures the CIP requirement in a natural way.

\section{Definition 2.5 (grounds equivalence)}

$$
\varphi \equiv_{G} \psi \text { iff } G \llbracket \varphi \rrbracket=G \llbracket \psi \rrbracket
$$

Obviously, ' $\equiv_{G}$ ' is an equivalence relation on meanings. An easy inspection of the proof of the context incorporation theorem shows that the meanings of the context-incorporated counterparts of contextually restricted quantified sentences are grounds equivalent.

\subsection{Harmony of the contextual domain restriction rules}

Prior's famous attack on the PTS-programme in (Prior 1960) produced a connective with an $I$-rule of disjunction and an E-rule of conjunction, that trivialized ' $\vdash$ ' so that $\varphi \vdash \psi$ for every $\varphi$ and $\psi$. As became evident since that attack, not every set of $I / E$-rules may qualify as conferring meaning. One of the prevailing criteria for an ND-system to qualify as conferring meaning is that of harmony, advocated by Dummett, Prawitz, Tennant and many others, requiring a balance between the $I$-rules and $E$-rules of every connective, in that neither group is either too weak or too strong w.r.t. the other group. Clearly, Prior's connective fails this condition. Two main ways to capture the informal notion of harmony were proposed in the literature.

Intrinsic harmony: According to this view of harmony, there is a requirement that every maximal formula $\varphi$ in a derivation, one that is a conclusion of an $I$-rule and the major premise of an $E$-rule (both of the main operator of $\varphi$ ), be eliminable, producing an equivalent derivation (with the same assumptions and same conclusion). The process of eliminating such a maximal formula is known as (proof) reduction, 
and underlies Prawitz's normalization procedure (Prawitz 1965). ${ }^{9}$ Reductions show that nothing is gained by introducing and immediately eliminating. The gain here does not refer to efficiency (mostly lengths) of derivations, but to the ability to draw additional conclusion. ${ }^{10}$ In a balanced system, any conclusion drawn by means of a maximal formula can be drawn without it, as shown by the reduction. Failing this condition shows that the $I$-rule is too strong (or the $E$-rule too weak). The second facet of the balance between $I / E$-rules is that of stability, excluding a situation in which the E-rules are too weak w.r.t. the $I$ rules. I will ignore this issue here.

Harmony in form: Under this view of harmony, the $E$-rules are required to have a specific form, known as general elimination (GE), allowing the derivation of an arbitrary conclusion using the premises of the $I$-rules as discharged assumptions. The standard rules $(V E)$ and $(\exists E)$ are of this form. GE-rules emerged independently of harmony, allowing a better correspondence between normal ND-derivations and CUT-free derivations in sequent-calculi (see, for example, SchroederHeister 1984; von Plato 2000, 2001). In (Francez and Dyckhoff 2012) a general procedure ${ }^{11}$ is presented for deriving harmoniously induced $G E$-rules from given I-rules, ensuring the availability of the reductions required by intrinsic harmony.

Below, I show the reductions for the rules for ' $t_{c}$ '.

Universal contextually restricted quantification:

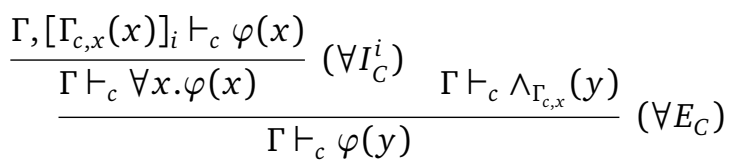

$$
\begin{aligned}
& \rightsquigarrow_{r} \Gamma[x:=y],\left[\Gamma_{c, x}(y)\right]_{i} \vdash_{c} \varphi(y)
\end{aligned}
$$

\footnotetext{
${ }^{9}$ Note that the presence of a reduction is less demanding than normalisation. The latter requires the finiteness of reduction sequences.

${ }^{10}$ Often, efficient derivation are not according to the meaning determined by $I$-rules. For example, if one first proves $\forall x . \varphi(x)$, and then derives (via $\forall E$ ) $\varphi(a), \varphi(b)$ etc., the derivations of the latter are shorter, but not according to meaning.

${ }^{11}$ Recently, some restrictions on the domain of applicability of this procedure have been realized, but they do not affect the current set-up.
} 
Note that since $x \notin$ free $(\Gamma), \Gamma[x:=y]=\Gamma$. The result follows by Proposition 2.1. A clearer depiction of the reduction uses $\mathscr{D}$ s.

$$
\begin{aligned}
& {\left[\wedge_{\Gamma_{c, x}}(x)\right]_{i}}
\end{aligned}
$$

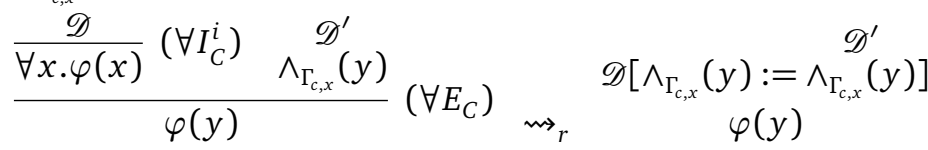

$\mathscr{D}^{\prime}$

where the substitution $\wedge_{\Gamma_{c, x}}(y):=\wedge_{\Gamma_{c, x}}(y)$ is the usual way composition of derivations is obtained, by replacing an assumption $\wedge_{\Gamma_{c}}(y)$ (a leaf in $\mathscr{D}$ ) with its given derivation in the second premise of $\left(\forall E_{C}\right)$.

The harmoniously induced $\left(\forall G E_{C}\right)$ is given below.

$$
\frac{\Gamma \vdash_{c} \forall x . \varphi(x) \quad \Gamma \vdash_{c} \wedge_{\Gamma_{c, x}}(y) \quad \Gamma,[\varphi(y)]_{i} \vdash_{c} \chi}{\Gamma \vdash_{c} \chi}\left(\forall G E_{C}^{i}\right), \quad y \text { fresh }
$$

Existential contextually restricted quantification: The reduction is the following.

$$
\begin{aligned}
& \begin{array}{ccc}
\mathscr{D}_{1} & \mathscr{D}_{2} \\
\frac{\varphi(y)}{\wedge_{\Gamma_{c, x}}(y)}\left(\exists I_{C}\right) & {[\varphi(z)]_{i},\left[\wedge_{\Gamma_{c, x}}(z)\right]_{j}} \\
\frac{\exists x \cdot \varphi(x)}{\chi} & \stackrel{\mathscr{D}}{\chi}\left(\exists E_{C}^{i, j}\right)
\end{array}
\end{aligned}
$$

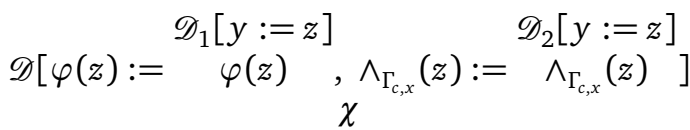

The ( $\exists E_{C}$ )-rule is in the $G E$-form to start with, thus harmonious in form too.

\subsection{Quantifier domain restriction in restricted quantification}

In order to make the subsequent presentation of QDR in NL more comprehensible, I exemplify the proof-theoretic approach by applying it first to a fragment $\mathrm{FOL}_{r q}$ of FOL that comes closer to the NL-fragment to be considered. The fragment is known as having restricted quantification (not to be confused with contextually restricted quantification, which is added on top of this). Quantified formulas in this fragment have the following form:

$$
\forall x . \varphi(x) \rightarrow \psi(x), \quad \exists x . \varphi(x) \wedge \psi(x)
$$


A proof-theoretic semantics for domain restriction

$$
\begin{gathered}
\frac{\Gamma, \xi \vdash \xi}{\Gamma,[\varphi(y)]_{i} \vdash \psi(y)} \\
\frac{\Gamma \vdash \forall x . \varphi(x) \rightarrow \psi(x)}{\left.\Gamma \vdash I^{i}\right)} \begin{array}{c}
\frac{\Gamma \vdash \varphi(y) \quad \Gamma \vdash \psi(y)}{\Gamma \vdash \exists x . \varphi(x) \wedge \psi(x)}(\exists I) \\
y \text { fresh for } \Gamma \text { in }(\forall I) .
\end{array} \\
\frac{\Gamma \vdash \forall x . \varphi(x) \rightarrow \psi(x) \quad \Gamma \vdash \varphi(y)}{\Gamma \vdash \psi(y)}(\forall E) \quad \frac{\Gamma \vdash \exists x . \varphi(x) \wedge \psi(x) \quad \Gamma,[\varphi(y)]_{i},[\psi(y)]_{j} \vdash \xi}{\Gamma \vdash \xi \quad y \text { fresh for } \Gamma, \xi \text { in }(\exists E) .}\left(\exists E^{i, j}\right)
\end{gathered}
$$

Figure 1: A natural-deduction proof system $N_{r q}$ for restricted quantification

The universal quantification can be read as 'everything which is $\varphi$ is $\psi$ ', and the existential quantification can be read as 'there exists something which is $\varphi$ that is $\psi$ '. That is, quantification is restricted to entities satisfying $\varphi$, to be called the restrictor. A more transparent syntax, closer to the natural language expression of quantification, would be

$$
\forall x: \varphi(x) . \psi(x), \quad \exists x: \varphi(x) \cdot \psi(x)
$$

The expression of (2.28) as (2.27) is known as Frege's translation, that has drawn criticism as a way to capture natural language quantification. For example, see (Ben-Yami 2006) and (Francez 2014b) for such a criticism. As I show in the next section, $\mathrm{FOL}_{r q}$-quantification reflects more directly natural language quantification.

The proof system is presented in Figure 1. I use $\Gamma \vdash \varphi$ in this subsection to indicate derivability of $\varphi$ from $\Gamma$ (in $N_{r q}$ ). A $G E$-rule for the universal quantifier, exhibiting harmony in form, is

$$
\begin{aligned}
& \frac{\Gamma \vdash \forall x . \varphi(x) \rightarrow \psi(x) \quad \Gamma \vdash \varphi(y) \quad \Gamma,[\psi(y)]_{i} \vdash \xi}{\Gamma \vdash \xi}\left(\forall G E^{i}\right) \\
& y \text { fresh }
\end{aligned}
$$

Next, I consider QDR in FOL ${ }_{r q}$. The observation is that the restrictor can be interpreted differently in different DR-contexts. Thus, the natural regimentation of (1.1) (cf. Example (2.1)) would again be

$$
\forall x . B(x) \rightarrow E(x)
$$

where ' $B(x)$ ' expresses $x$ is a bottle and ' $E(x)$ ' expresses $x$ is empty. Here, the restrictor $B(x)$ can have a contextually varying interpretation. 
The idea for the proof-theoretic representation of contextual meaning variation is as before, where for universal quantification a DR-context $c$ provides a contextual discharged assumption. The generated contextual restriction strengthens the restriction already present in the formula. The rules are shown below. ${ }^{12}$

$$
\begin{gathered}
\frac{\Gamma,[\varphi(x)]_{j},\left[\Gamma_{c, x}(x)\right]_{i} \vdash_{c} \psi(x)}{\Gamma \vdash_{c} \forall x . \varphi(x) \rightarrow \psi(x)}\left(\forall I_{C}^{i, j}\right), \quad x \notin \operatorname{free}(\Gamma) \\
\frac{\Gamma \vdash_{c} \forall x . \varphi(x) \rightarrow \psi(x) \quad \Gamma \vdash_{c} \varphi(y) \wedge \wedge_{\Gamma_{c, x}}(y)}{\Gamma \vdash_{c} \psi(y)}\left(\forall E_{C}\right) \\
\frac{\Gamma \vdash_{c} \varphi(y) \quad \Gamma \vdash_{c} \Gamma_{c, x}(y) \quad \Gamma \vdash \psi(y)}{\Gamma \vdash_{c} \exists x . \varphi(x) \wedge \psi(x)}\left(\exists I_{C}^{i}\right) \\
\frac{\Gamma \vdash_{c} \exists x . \varphi(x) \wedge \psi(x) \quad \Gamma,\left[\left(\varphi \wedge \wedge_{\Gamma_{c, x}} \wedge \psi\right)(y)\right]_{i} \vdash_{c} \chi}{\Gamma \vdash_{c} \chi}\left(\exists E^{i}\right)
\end{gathered}
$$

where $y \notin$ free $(\Gamma, \chi)$ in $(\exists E)$.

The same considerations as those for FOL show that (CIP) holds also for $\mathrm{FOL}_{r q}$.

$$
\begin{gathered}
\text { PROOF-THEORETIC SEMANTICS } \\
\text { FOR QUANTIFIER DOMAIN RESTRICTION } \\
\text { IN A FRAGMENT OF ENGLISH }
\end{gathered}
$$

In this section, I present a PTS for QDR in its more natural setting, within an extensional fragment of English. A PTS for such a fragment (without considering QDR) is provided in (Francez and Dyckhoff 2010).

For self-containment of the paper, this semantics is reviewed below.

\subsection{Review of the proof-theoretic semantics for sentences}

I present the fragment and its associated proof system in two stages. First, a core fragment is presented, extended in a second stage with relative clauses and intersective adjectives.

\footnotetext{
${ }^{12}$ The notation $\varphi(y) \wedge \wedge_{\Gamma_{c, x}}(y)$ means the conjunction of $\varphi(y)$ with the conjunction of the context formulas in $\Gamma_{c, x}$ applied to $y$.
} 
The core fragment $E_{0}^{+}$of English consists of sentences headed by (extensional) intransitive and transitive verbs, and determiner phrases $(d p)$ with a (singular, count) noun and a determiner. In addition, there is the copula. This is a typical fragment of many NLs, syntactically focusing on subcategorization, and semantically focusing on predication and quantification. Some typical sentences are listed below.

every/some girl smiles/is a student/loves some boy

I omit here proper names that do appear in the detailed presentation of sentential meanings (Francez and Dyckhoff 2010). Note the absence of negative determiners like no (hence the superscript ' + '), which are treated in (Francez and Ben-Avi 2014), involving technicalities orthogonal to QDR. Expressions such as every girl, some boy are $d p s$.

The PTS is based on a core dedicated, meaning-conferring naturaldeduction proof system $N_{0}^{+}$with $I / E$-rules presented in Figure 2 . The proof system is formulated over the language $L_{0}^{+}$, slightly extending $E_{0}^{+}$ and disambiguating ambiguous $E_{0}^{+}$sentences. Meta-variables $X$ schematize nouns, $P$ over intransitive verbs and $R$ over transitive verbs. Metavariable $S$ ranges over sentences, and boldface lower-case $\mathbf{j}$, $\mathbf{k}$, etc., range over $\mathscr{P}$, a denumerable set of (individual) parameters, artefacts of the proof system (not used to make assertions). Syntactically, a parameter in $L_{0}^{+}$is also regarded as a $d p$. If a parameter occurs in $S$ in some position, $S$ is a pseudo-sentence, and if all $d p s$ in $S$ are parameters, the pseudo-sentence $S$ is ground. The ground pseudo-sentences play the role of atomic sentences, and their meaning is assumed given, externally to the ND proof system. The latter defines sentential meanings of non-ground pseudo-sentences (and, in particular, $E_{0}^{+}$-sentences), relative to the given meanings of ground pseudo-sentences.

In contrast to logic, where the introduced operator by an $I$-rule is always the (unique) main operator, in $E_{0}^{+}$sentences there is no such main operator: every position that can be filled with a $d p$ is a locus of introduction (of the quantifier corresponding to the determiner of the introduced $d p$ ). This is a major source of ambiguity in $E_{0}^{+}$, known as quantifier-scope ambiguity. The way ambiguity is treated is recapitulated briefly below. For any $d p$-expression $D$ having a quantifier, I use the notation $S\left[(D)_{n}\right]$ to refer to a sentence $S$ having a designated 
Figure 2:

The meta-rules for $N_{0}^{+}$

$$
\begin{gathered}
\overline{\Gamma, S \vdash S} \quad(A x) \\
\frac{\Gamma,[\mathbf{j} \text { isa } X]_{i} \vdash S[\mathbf{j}]}{\Gamma \vdash S\left[(\text { every } X)_{r(S[\mathbf{j}])+1}\right]}\left(e I^{i}\right) \\
\frac{\Gamma \vdash \mathbf{j} \text { isa } X \quad \Gamma \vdash S[\mathbf{j}]}{\Gamma \vdash S\left[(\text { some } X)_{r(S[\mathbf{j}])+1}\right]}(s I) \\
\frac{\Gamma \vdash S\left[(\text { every } X)_{r(S[\mathbf{j}])+1}\right] \quad \Gamma \vdash \mathbf{j} \text { isa } X \quad \Gamma,[S[\mathbf{j}]]_{i} \vdash S^{\prime}}{\Gamma \vdash S^{\prime}}\left(e E^{i}\right) \\
\frac{\Gamma \vdash S\left[(\text { some } X)_{r(S[\mathbf{j}])+1}\right] \quad \Gamma,[\mathbf{j} \text { isa } X]_{j},[S[\mathbf{j}]]_{i} \vdash S^{\prime}}{\Gamma \vdash S^{\prime}}\left(s E^{i, j}\right)
\end{gathered}
$$

where $\mathbf{j}$ is fresh for $\Gamma, S[$ every $X]$ in $(e I)$, and for $\Gamma, S[$ some $X], S^{\prime}$ in $(s E)$.

position filled by $D$, where $n$ is the scope level (sl) of the quantifier in $D$. In case $D$ has no quantifier (i.e., it is a parameter), $s l=0$. The higher $s l$, the higher the scope. For example, $S\left[(\text { every } X)_{1}\right]$ schematizes a sentence $S$ with a designated occurrence of every $X$ of the lowest scope. An example of a higher scope is $S\left[(\text { some } X)_{2}\right]$, having some $X$ in the higher scope, like in the object wide-scope reading of (every $X)_{1}$ loves (some $\left.Y\right)_{2}$. I use the conventions that within a rule, both $S\left[D_{1}\right]$ and $S\left[D_{2}\right]$ refer to the same designated position in $S$, and when the $s l$ can be unambiguously determined it is omitted. I use $r(S)$ to indicate the rank of $S$, the highest $s l$ on a $d p$ within $S$. Note that for a ground $S, r(S)=0$.

Recall that in a rule, the notation $[\cdots]_{i}$ indicates an assumption discharged by an application of that rule. The indices of the assumptions discharged by a rule appear as superscripts on the rule name. The usual notion of (tree-shaped) derivation is assumed. I again use $\mathscr{D}$ to range over derivations, where $\mathscr{D}^{\Gamma \vdash S}$ is a derivation of sentence $S$ from assumptions $\Gamma$. I use $\Gamma, S$ for extending $\Gamma$ with a sentence $S$. A more detailed explanation of the rules is presented in (Francez and Dyckhoff 2010). However, it is evident that all quantification in the fragment is restricted. In addition to this restriction I will add QDR in the next section.

The following is a convenient derived E-rule, that will be used to shorten derivations.

$$
\frac{\Gamma \vdash S\left[(\text { every } X)_{r(S[\mathbf{j}])+1}\right] \quad \Gamma \vdash \mathbf{j} \text { isa } X}{\Gamma \vdash S[\mathbf{j}]}(e \hat{E})
$$


Below is an example derivation establishing some $U$ isa $X$, (every $X)_{2} R(\text { some } Y)_{1}$, every $Y$ isa $Z \vdash(\text { some } U)_{1} R$ (some $\left.Z\right)_{2}$.

$\mathscr{D}_{1}$

Let (some $U)_{2} R$ (some $\left.Z\right)_{1}$ and (some $\left.U\right)_{1} R$ (some $\left.Y\right)_{2}$ be the following two sub-derivations.

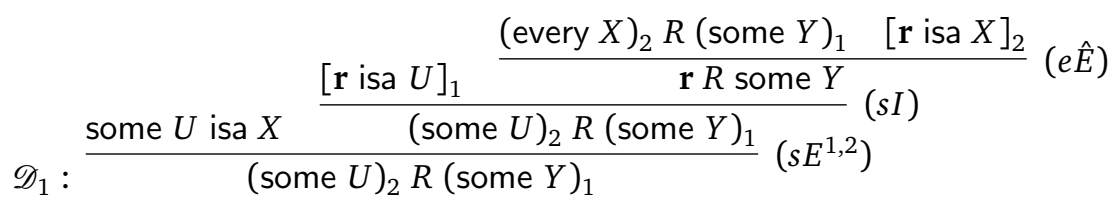

$$
\begin{aligned}
& \mathscr{D}_{2}: \frac{[\text { some } U R \mathbf{j}]_{3}}{(\text { some } U)_{1} R(\text { some } Z)_{2}}(s I)
\end{aligned}
$$

The whole derivation combines the two sub-derivations by

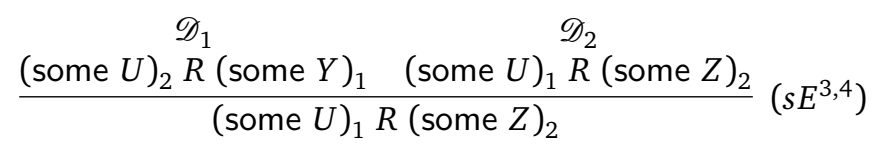

For a derivation $\mathscr{D}$ of $S$, its root is given by $\rho(\mathscr{D})=S$. This function is extended to collections of derivations $\Delta$ by $\rho(\Delta)=\{\rho(\mathscr{D}) \mid \mathscr{D} \in$ $\Delta\}$, and further extended to contextualized functions $\mathscr{F}$ by $\rho(\mathscr{F})=$ $\cup_{\Gamma} \rho(\mathscr{F}(\Gamma))$.

In order to understand better the PTS of $E_{0}^{+}$, consider one of its well-known features: quantifier scope ambiguity. The following $E_{0}^{+}$ sentences are usually attributed to two readings each, with the following FOL-expressions of their respective truth-conditions in modeltheoretic semantics.

$$
\begin{aligned}
& \text { Every girl loves some boy } \\
& \text { Some girl loves every boy }
\end{aligned}
$$

Consider sentence (3.34).

Subject wide-scope (sws): $\forall x \cdot \operatorname{girl}(x) \rightarrow \exists y \cdot \operatorname{boy}(y) \wedge \operatorname{love}(x, y)$

Subject narrow-scope (sns): $\exists y \cdot \operatorname{boy}(y) \wedge \forall x \cdot \operatorname{girl}(x) \rightarrow \operatorname{love}(x, y)$

In the proposed PTS, the difference in meanings reflects itself by the two readings having different uses of the grounds for assertion. This is 
manifested in derivations by different orders of introduction of the subject and object $d p s$. Following Moss (2010), I disambiguate ambiguous sentences taking part in derivations.

\section{Subject wide-scope (sws):}

$$
\frac{\frac{[\mathbf{r} \text { isa girl }]_{i}}{\frac{\mathscr{D}_{1}}{\mathbf{r} \text { loves } \mathbf{j}}} \frac{\mathscr{D}_{2}}{\mathbf{\mathbf { j } \text { loves boy } ( \text { some boy } ) _ { 1 }}}(s I)}{\text { (every girl) } \left.)_{2} \text { loves (some boy) }\right)_{1}}\left(e I^{i}\right)
$$

Subject narrow-scope (sns):

$$
\frac{\frac{[\mathbf{r} \text { isa girl }]_{i}}{\mathscr{D}_{1}}}{\frac{\mathbf{r} \text { loves } \mathbf{j}}{(\text { every girl })_{1} \text { loves } \mathbf{j}}\left(e I^{i}\right) \frac{\mathscr{D}_{2}}{\mathbf{j} \text { isa boy }}}(s I)
$$

Note that there is no way to introduce a $d p$ with a narrow-scope where the $d p$ with the wider-scope has already been introduced. In the $N_{0}^{+}$ calculus, only disambiguated sentences participate.

Relative clauses and intersective adjectives

I next add relative clauses to the fragment, followed by intersective adjectives. This fragment transcends the locality of subcategorization in $E_{0}^{+}$, in having long-distance dependencies. It also has unbounded number of adjectival modifications. I refer to this (still positive) fragment as $E_{1}^{+}$. Note that, in contrast to $E_{0}^{+}, E_{1}^{+}$is infinite. Typical sentences include the following.

$$
\text { every some/boy loves every/some girl }
$$

who( $m)$ smiles/loves every/some flower/whom some girl loves

$$
\text { every/some girl is a girl who loves every/some boy }
$$

some boy loves every/some girl who loves every boy who smiles

$$
\text { (nested relative clause) }
$$

So, girl who smiles and girl who loves every boy are compound nouns. I treat somewhat loosely the issue of the case of the relative pronoun, 
in the form of who(m), abbreviating either who or whom, as the case requires. I extend the notation with $S[-]$, which denotes, for $S$ including a parameter in some distinguished position, the result of removing that parameter, leaving that position unoccupied. Examples are loves every girl (a parameter removed from subject position in $\mathbf{j}$ loves every girl), and every girl loves (a parameter removed from object position in every girl loves $\mathbf{k}$ ).

The corresponding ND-system $N_{1}^{+}$extends $N_{0}^{+}$by adding the following $I / E$-rules.

$$
\begin{gathered}
\frac{\Gamma \vdash \mathbf{j} \text { isa } X \quad \Gamma \vdash S[\mathbf{j}]}{\Gamma \vdash \mathbf{j} \text { isa } X \text { who } S[-]}(\text { rell }) \\
\frac{\Gamma \vdash \mathbf{j} \text { isa } X \text { who } S[-] \quad \Gamma,[\mathbf{j} \text { isa } X]_{i},[S[\mathbf{j}]]_{j} \vdash S^{\prime}}{\Gamma \vdash S^{\prime}}\left(\text { relE }^{i, j}\right)
\end{gathered}
$$

The simplified derived $E$-rules are:

$$
\frac{\Gamma \vdash \mathbf{j} \text { isa } X \text { who } S[-]}{\Gamma \vdash \mathbf{j} \text { isa } X}(\operatorname{rel} \hat{E})_{1} \quad \frac{\Gamma \vdash \mathbf{j} \text { isa } X \text { who } S[-]}{\Gamma \vdash S[\mathbf{j}]}(\operatorname{rel} \hat{E})_{2}
$$

The familiar conjunctive behavior of relative clauses is exhibited here by its rules, resembling the rules for logical conjunction.

As an example of a derivation in this fragment, consider

$$
\text { some girl who smiles sings } \vdash_{N_{1}^{+}} \text {some girl sings }
$$

exhibiting the upward monotonicity of some in its first argument.

$$
\frac{\text { some } X \text { who } P_{1} P_{2} \quad \frac{\left[\mathbf{r} \text { isa } X \text { who } P_{1}\right]_{1}}{\mathbf{r} \text { isa } X}(r e l \hat{E})_{1} \quad\left[\mathbf{r} P_{2}\right]_{2}}{\text { some } X P_{2}}(s I)
$$

Finally, I augment $E_{1}^{+}$with sentences containing adjectives, schematized by $A$. I consider here only what is known in model-theoretic semantics as intersective adjectives. Typical sentences are:

Some girl is a beautiful girl/clever beautiful girl/clever beautiful red-headed girl

every/some beautiful girl smiles 
every/some beautiful girl loves every/some clever boy

A noun preceded by an adjective is again a (compound) noun (the syntax is treated more precisely once the grammar is presented, as in Francez et al. 2010). Denote this extension still by $E_{1}^{+}$. Recall that in the $N_{1}^{+}$rules, the noun schematization should be taken over compound nouns too. Note that I augment $N_{1}^{+}$with the following ND-rules for adjectives.

$$
\begin{gathered}
\frac{\Gamma \vdash \mathbf{j} \text { isa } X \quad \Gamma \vdash \mathbf{j} \text { is } A}{\Gamma \vdash \mathbf{j} \text { isa } A X}(\operatorname{adj} I) \\
\frac{\Gamma \vdash \mathbf{j} \text { isa } A X \quad \Gamma,[\mathbf{j} \text { isa } X]_{1},[\mathbf{j} \text { is } A]_{2} \vdash S^{\prime}}{\Gamma \vdash S^{\prime}}\left(\operatorname{adj} E^{1,2}\right)
\end{gathered}
$$

Again, the following derived E-rules are used to shorten presentations of example derivations.

$$
\frac{\Gamma \vdash \mathbf{j} \text { isa } A X}{\Gamma \vdash \mathbf{j} \text { isa } X}\left(\operatorname{adj} \hat{E}_{1}\right) \frac{\Gamma \vdash \mathbf{j} \text { isa } A X}{\Gamma \vdash \mathbf{j} \text { is } A}\left(\operatorname{adj} \hat{E}_{2}\right)
$$

Note that the intersectivity here is manifested by the rules themselves (embodying an invisible conjunctive operator) at the sentential level. These rules induce intersectivity as a lexical property of (some) adjectives by the way lexical meanings are extracted from sentential meanings, as shown in (Francez et al. 2010).

The following sequent, the corresponding entailment of which is often taken as the definition of intersective adjectives, is derivable in $N_{1}^{+}$:

$$
\mathbf{j} \text { isa } A X, \mathbf{j} \text { isa } Y \vdash \mathbf{j} \text { isa } A Y
$$

as shown by

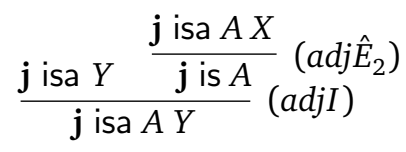

As an example of derivations using the rules for adjectives, consider the following derivation for

$$
\mathbf{j} \text { loves every girl } \vdash \mathbf{j} \text { loves every beautiful girl }
$$

In model-theoretic semantics terminology, the corresponding entailment is a witness to the downward monotonicity of the meaning of every 
in its second argument. I use an obvious schematization.

$$
\frac{\mathbf{j} R \text { every } Y \frac{[\mathbf{r} \text { isa } A Y]_{1}}{\mathbf{r} \text { isa } Y}(a \operatorname{dj} \hat{E})}{\frac{\mathbf{j} R \mathbf{r}}{\mathbf{j} R \text { every } A Y}\left(e I^{1}\right)}
$$

Under this definition of the meaning of intersective adjectives, such adjectives are also extensional, in the sense of satisfying the following entailment:

$$
\text { every } X \text { isa } Y \vdash \text { every } A X \text { isa } A Y
$$

as shown by the following derivation:

$$
\frac{\text { every } X \text { isa } Y}{\frac{[\mathbf{j} \text { isa } A X]_{1}}{\mathbf{j} \text { isa } X}\left(\operatorname{adj} \hat{E}_{1}\right)}(e \hat{E}) \quad \frac{[\mathbf{j} \text { isa } A X]_{1}}{\mathbf{j} \text { is } A}\left(\operatorname{adj} \hat{E}_{2}\right)
$$

The proof of harmony of $N_{1}^{+}$can be found in (Francez and Dyckhoff 2010) and is not repeated here.

Sentential meanings

Again, a derivation is canonical if it essentially ends with an application of an $I$-rule; I use $\vdash^{c}$ for canonical derivability, denote by $\llbracket S \rrbracket_{\Gamma}^{c}$ the collection of canonical derivations of $S$ from $\Gamma$, and by $\llbracket S \rrbracket_{\Gamma}^{*}$ the collection of all derivations of $S$ from $\Gamma$.

Those proof-theoretic collections are used to define meanings. Note that these are strictly proof-theoretic denotations, independent from any notion of a model, entities, and the like.

\section{Definition 3.6 (PTS-meaning, semantic values)}

1. For a non-ground $S \in L_{1}^{+}$, its (reified) meaning (also referred to as its contributed semantic value) is given by $\llbracket S \rrbracket \stackrel{\text { df. }}{=} \lambda \Gamma$. $\llbracket S \rrbracket_{\Gamma}^{c}[=$ $\left.\lambda \Gamma \cdot\left\{\mathscr{D}^{\Gamma{ }^{c}} \mathrm{~S}\right\}\right]$.

Recall that for a ground $S$, $\llbracket S \rrbracket$ is assumed given. The meaning of non-ground pseudo-sentences (and $E_{0}^{+}$-sentences in particular) is defined relative to the given meanings of ground pseudosentences. 
2. For an arbirary $S \in L_{1}^{+}$, its contributing semantic value is given by $\llbracket S \rrbracket^{*} \stackrel{\text { df. }}{=} \lambda \Gamma \cdot \llbracket S \rrbracket_{\Gamma}^{*}$.

This distinction corresponds to the one that Dummett (1993, p. 48) introduced between assertoric content and ingredient sense. The content of an (affirmative) sentence $S$ is the meaning of $S$ in isolation, on its own. The ingredient sense of $S$ is what $S$ contributes to the meaning of any $S^{\prime}$ in which $S$ occurs as a sub-expression, a component. This distinction is propagated to sub-sentential phrases as well. I will be concerned here with the contents of sentences only.

The main characteristic of this definition of (proof-theoretic) meaning is the notion of entailment it induces. A more comprehensive discussion can be found in (Francez 2014c).

By defining sentential meanings in this way, I do not allude to any logical form of the sentence differing from its surface form. In accordance with many views in philosophy of language, every derivation in the meaning of a sentence $S$ can be viewed as providing $G \llbracket S \rrbracket$, grounds for asserting $S$. Definition (2.4) is adapted to the current fragment.

Definition 3.7 (grounds for assertion - NL) For $S \in E_{1}^{+}, G \llbracket S \rrbracket \stackrel{\text { df. }}{=}$ $\left\{\Gamma \mid \Gamma \vdash^{c} S\right\}$, where $\Gamma$ consists of $E_{1}^{+}$-sentences only. Parameters are not observable in grounds of assertion.

The refinement of the (reified) sentential meanings via ' $\equiv_{G}$ ' is used here too, for the CIP (see below). A more comprehensive discussion of extensions of the fragment and some technicalities accompany the original presentation of the PTS in (Francez and Dyckhoff 2010).

In this section, I develop the proof-theoretic semantics for QDR in setting of the natural language fragment $E_{1}^{+}$. This setting is more suitable for that task than that of FOL and $\mathrm{FOL}_{r q}$, that were considered for ease of presentation of the approach, being more familiar to most readers than the dedicated $N_{1}^{+}$.

Definition 3.8 (NLDR-context) An NLDR-context (NL domain restricting context) $c$ is a finite collection $\Gamma_{c}$ of pseudo-sentences with one parameter only, where $\Gamma_{c, j}$ is the sub-collection with the parameter $\mathbf{j} .{ }^{13}$ Let $C N L$ (NL contexts) be the collection of all NLDR-contexts.

${ }^{13}$ For simplicity, I assume this sub-collection is a singleton. 
The NLDR-contexts $\Gamma_{c, \mathbf{j}}(\mathbf{j})$ can be of one of the following forms: $\mathbf{j}$ isa $X$ ( $X$ is a noun), $\mathbf{j}$ is $A$ (where $A$ is an adjective) or $\mathbf{j} P$ (where $P$ is a verb phrase). Note that compound contextual restrictions can also be imposed, as, for example, in $\Gamma_{c, \mathbf{j}}=\mathbf{j}$ isa man whom every girl loves. Since the fragment $E_{1}^{+}$has only modification by means of (intersective) adjectives and relative clauses, all the examples will be restricted to such modification. Extensions, for example, to incorporate preposition phrases, are not an obstacle in principle, but none have been proposed yet.

Restricting universal quantification: Again, an NLDR-context $c$ provides a discharged assumption for imposing its restriction.

$$
\begin{gathered}
\frac{\Gamma,[\mathbf{j} \text { isa } X]_{i},\left[\Gamma_{c, \mathbf{j}}(\mathbf{j})\right]_{j} \vdash_{c} S[\mathbf{j}]}{\Gamma \vdash_{c} S\left[(\text { every } X)_{r(S[\mathbf{j}])+1}\right]}\left(e I_{C N L}^{i, j}\right), \mathbf{j} \text { fresh for } \Gamma \\
\frac{\Gamma \vdash_{c} S\left[(\text { every } X)_{r(S[\mathbf{j}])+1}\right]}{\Gamma \vdash_{c} \mathbf{k} \text { isa } X} \quad \Gamma \vdash_{c} \Gamma_{c, \mathbf{j}}(\mathbf{k}) \quad \Gamma,[S[\mathbf{k}]]_{i} \vdash_{c} S^{\prime} \\
\Gamma \vdash_{c} S^{\prime}
\end{gathered}
$$

Again, a family of $I / E$-rules is employed, for all possible NLDRcontexts.

Example 3.4 Below is a derivation establishing

every Italian woman smiles, every woman who smiles is beautiful

$$
\vdash_{c} \text { every woman is beautiful }
$$

in an NLDR-context $c$ with $\Gamma_{c, \mathbf{k}}(\mathbf{k})=\mathbf{k}$ is Italian, intended to restrict the universal quantification on women to a universal quantification on Italian women. The observant reader will notice that Example 2.2 is a regimentation of this example in FOL. Since there is no quantifier scope ambiguity involved in this example, I omit in the derivation the scope level indicator to avoid notational clutter. Also, for typographical reasons, I abbreviate in the derivation Woman, Italian, Beautiful and Smiles to W, I, B and S, respectively. 
$\frac{[\mathbf{k} \text { isa W }]_{1}}{\frac{[\mathbf{k} \text { is }]_{2}[\mathbf{k} \text { isa W }]_{1}}{\mathbf{k} \text { isa I W }}(\operatorname{adjI}) \text { every I W S }}(e \hat{E})$

It is interesting to note that QDR holds, no matter which scope level the restricted quantifier is in.

Example 3.5 Consider the following scope variants of

some man admires every actress

In the NLDR-context $c$ with $\Gamma_{c, \mathbf{k}}=\mathbf{k}$ is Italian, intended to restrict the universal quantification on actresses to universal quantification on Italian actresses. I use the abbreviations $P$ for philosopher, $M$ for man, $I$ for Italian, $A$ for actress, $a d m$ for admires and $S$ for smart, to show that

some $P$ isa $M$, every $P$ is $S$, every $S M$ adm every $I A$

$$
\vdash_{c} \text { some } M \text { adm every } A
$$

under both scope variants of the conclusion. For typographical reasons, the derivations are presented with a common sub-derivation $\mathscr{D}$ factored out.

$$
\begin{aligned}
& \frac{[\mathbf{j} \text { isa } M]_{1} \frac{[\mathbf{j} \text { isa } P]_{2} \text { every } P \text { is } S}{\mathbf{j} \text { is } S}}{\mathbf{j} \text { isa } S M}(e \hat{E}) \\
& \mathscr{D}= \\
& \text { j adm every I } A
\end{aligned}
$$

Subject wide scope: The derivation is (with obvious abbreviations and $\Gamma$ omitted):

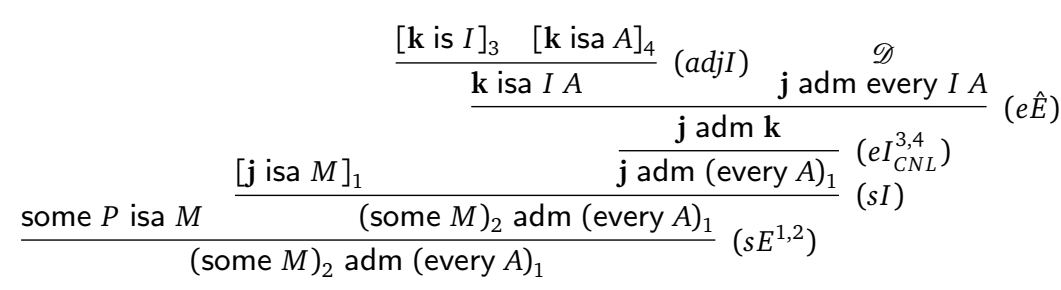


Object wide scope: The derivation is

$$
\frac{\text { some } P \text { isa } M \frac{[\mathbf{k} \text { is } I]_{3}[\mathbf{k} \text { isa } A]_{4}}{\mathbf{k} \text { isa } I A}(\operatorname{adjI}) \mathbf{j} \text { adm every } I A}{\mathscr{j} \text { isa } M]_{1}}(e \hat{E})
$$

Example 3.6 The following example from (Stanley and Szabó 2000) is pointed out as being difficult for MTS-handling, as it seemingly requires context-shift during meaning evaluation.

$$
\text { every sailor waved to every sailor }
$$

where the context imposes the restriction that the quantification in the subject is restricted to one kind of sailors, say sailors on the ship, while the object quantification is restricted, say, to sailors on the shore. Under the current approach, such examples pose no problem whatsoever. Suppose that $\mathbf{j}$ is the parameter used to introduce every sailor in the subject, while $\mathbf{k}$ is the parameter used to introduce every sailor in the object (where both scope relations are equivalent). Then, all we have to do is consider a context $c_{\text {sailors }}$, with $\Gamma_{c_{\text {sailors }}, j}=\mathbf{j}$ is - on - the-ship, and $\Gamma_{c_{\text {sailors }}, k}=\mathbf{k}$ is - on - the - shore. No context shift is involved. As a full derivation is somewhat lengthy, I skip the details.

\section{Restricting existential quantification:}

$$
\begin{gathered}
\frac{\left.\Gamma \vdash \mathbf{j} \text { isa } X \quad \Gamma \vdash \Gamma_{c, \mathbf{j}} \mathbf{j}\right) \quad \Gamma \vdash S[\mathbf{j}]}{\Gamma \vdash_{c} S\left[(\operatorname{some} X)_{r(S[\mathbf{j}])+1}\right]}\left(s I_{C N L}\right) \\
\frac{\Gamma \vdash S\left[(\text { some } X)_{r(S[\mathbf{j}])+1}\right] \quad \Gamma,[\mathbf{k} \text { isa } X]_{i},\left[\Gamma_{c, \mathbf{j}}(\mathbf{k})\right]_{j},[S[\mathbf{k}]]_{k} \vdash S^{\prime}}{\Gamma \vdash S^{\prime}}\left(s E_{C N L}^{i, j, k}\right)
\end{gathered}
$$

where $\mathbf{k}$ is fresh for $\Gamma, S[$ some $X], S^{\prime}$ in $\left(s E_{C N L}\right)$.

The reductions needed to show the harmony of the $C N L$-rules are very similar to those for the regular rules (shown in Francez and Dyckhoff 2010) and are omitted.

Next, I show how the CIP is satisfied for $E_{1}^{+}$. Note that the fragment includes neither implication nor conjunction (on the sentential level). 
To express the CIP effect, I use the following notation. For $S[(q X)]$ (with $q$ either every or some), let $S_{\Gamma_{c, j}}$ be defined as

$$
S_{\Gamma_{c, j}}=\left\{\begin{array}{cc}
S[(q X \text { who isa } Y)] & \Gamma_{c, \mathbf{j}}(\mathbf{j})=\mathbf{j} \text { isa } Y \\
S[(q A X)] & \Gamma_{c, \mathbf{j}}(\mathbf{j})=\mathbf{j} \text { is } A \\
S[(q X \text { who } P)] & \Gamma_{c, \mathbf{j}}(\mathbf{j})=\mathbf{j} P
\end{array}\right.
$$

Theorem 3.2 ( $E_{1}^{+}$context incorporation)

$$
\Gamma \vdash_{c} S[(q X)] \text { iff } \Gamma \vdash S_{\Gamma_{c, j}}
$$

Proof: I will show only the proof of the first case, for $q=$ every; all other cases are similar. To simplify, I also omit the scope indications.

1. Asume $\Gamma \vdash_{c} S[($ every $X)]$, where $\Gamma_{c, \mathbf{j}}=\mathbf{j}$ isa $Y$. So, the derivation ends with (omitting scope indication)

$$
\frac{\Gamma,[\mathbf{j} \text { isa } X]_{i},[\mathbf{j} \text { isa } Y]_{j} \vdash_{c} S[\mathbf{j}]}{\Gamma \vdash_{c} S[(\text { every } X)]}\left(e I_{C N L}^{i, j}\right), \mathbf{j} \text { fresh for } \Gamma
$$

Therefore, the following derivation can be formed, where the induction hypothesis on the premise uses ' $\vdash$ ' instead of ' $\vdash$ '.

$$
\frac{\Gamma,[\mathbf{j} \text { isa } X \text { who isa } Y]_{i}}{\frac{\Gamma \vdash \mathbf{j} \text { isa } X}{(r e l E)} \frac{\Gamma,[\mathbf{j} \text { isa } X \text { who isa } Y]_{i}}{\Gamma \vdash \mathbf{j} \text { isa } Y}(\text { ass.) }}(\text { relE) }
$$

2. Assume $\Gamma \vdash S[$ (every $X$ who isa $Y)]$. The derivation (again, omitting scope indication) ends with

$$
\frac{\Gamma,[\mathbf{j} \text { isa } X \text { who isa } Y]_{i} \vdash S[\mathbf{j}]}{\Gamma \vdash S[(\text { every } X \text { who isa } \mathrm{Y})]}\left(e I^{i}\right)
$$

Let $\Gamma_{c, \mathbf{j}}=\mathbf{j}$ isa $Y$. Therefore, the following derivation can be formed:

$$
\begin{gathered}
\frac{[\Gamma \vdash \mathbf{j} \text { isa } X]_{i} \quad[\Gamma \vdash \mathbf{j} \text { isa } Y]_{j}}{\Gamma \vdash \mathbf{j} \text { isa } X \text { who isa } Y}(\text { relI }) \\
\frac{S[\mathbf{j}]}{\left.\Gamma \vdash_{c} S[\text { (every } X)\right]}\left(e I_{C N L}^{i, j}\right)
\end{gathered}
$$


Once again, by inspecting the rules, we obtain that

$$
\llbracket S[(q X)] \rrbracket_{c}=\llbracket S_{\Gamma_{c, j}} \rrbracket
$$

validating the CIP. Note again the correspondence between derivations, where a use of $\left(q I_{N L C}\right)$ is associated with $(q I)$ followed by (rel $\left.I\right)$ (or by (Adj $I$ ) in some of the cases), and similarly for the $E$-rules.

\section{GONGLUSIONS}

The paper introduces proof-theoretic semantics for contextual domain restriction as an alternative to the model-theoretic meaning generally found in the literature. In addition to providing yet another example for the feasibility of PTS for natural language meanings, the paper points to an advantage (in my opinion) of the PTS approach to QDR over the MTS approach; namely, the CIP principle, by which every contextually restricted quantified sentence has the same meaning as a context-independent variant thereof, where the contextual restriction is incorporated as a phrase in the sentence. Thus, no equivalent of intersection with arbitrary subsets of the quantification domain, not being the denotation (in the model) of any NL expression, is involved. Some other advantages related to multiple quantification have also been shown. In particular, both facets of the QDR-problem pointed out by Stanley and Szabó (2000), namely the descriptive and the fundamental, are treated, in contrast to the MTS discussion in the literature, which usually evades the latter.

As observed by one of the referees of this paper, an important phenomenon related to contextual meaning variation, namely, pronominal binding, is not covered by the proposed PTS. The reason is that currently the fragment for which a PTS has been proposed does not include pronouns at all. I consider this to be a topic of further work, both extending the fragment with pronouns and investigating the impact of such an extension of the general contextual QDR-problem.

The approach was also exemplified in two variants of FOL (firstorder logic). The current interface between the contextual restriction and the sentential derivation is through the name of the variable involved (or in the NL case, through the parameter). This might seem somewhat ad hoc, and a more transparent binding of contextual restrictions and the corresponding quantifiers should be sought. 


\section{Nissim Francez}

\section{REFERENGES}

Hanoch BEN-YAMI (2006), A critique of Frege on common nouns, Ratio, 19(2):148-155.

Robert B. BRANDOM (2000), Articulating reasons, Harvard University Press, Cambridge, MA.

Michael DumMETT (1993), The logical basis of metaphysics, Harvard University Press, Cambridge, MA, USA, hard copy 1991.

Nissim FRANCEZ (2014a), The granularity of meaning in proof-theoretic semantics, in Nicholas ASHER and Sergei SOLOVIEW, editors, Proceedings of the 8th International Conference on Logical Aspects of Computational Linguistics (LACL), Toulouse, France, June 2014, volume 8535 of LNCS, pp. 96-106, Springer Verlag, Berlin/Heidelberg, Germany.

Nissim FRANCEZ (2014b), A logic inspired by natural language: quantifiers as subnectors, Journal of Philosophical Logic, doi:10.1007/s10992-014-9312-z.

Nissim FRANCEZ (2014c), Views of proof-theoretic semantics: Reified proof-theoretic meanings, Journal of Computational Logic, special issue in honour of Roy Dyckhoff, doi:10.1093/logcom/exu035.

Nissim FrANCEZ and Gilad BEN-Avi (2011), Proof-theoretic semantic values for logical operators, Review of Symbolic Logic, 4(3):337-485.

Nissim FRANCEZ and Gilad BEN-AVI (2014), A proof-theoretic reconstruction of generalized quantifiers, Journal of Semantics, doi:10.1093/jos/ffu001.

Nissim FrANCEZ and Roy DYCKHOFF (2010), Proof-theoretic semantics for a natural language fragment, Linguistics and Philosophy, 33(6):447-477.

Nissim FRANCEZ and Roy DYCKHOFF (2012), A note on harmony, Journal of Philosophical Logic, 41(3):613-628.

Nissim Francez, Roy Dyckhoff, and Gilad Ben-Avi (2010), Proof-theoretic semantics for subsentential phrases, Studia Logica 94, pp. 381-401.

Nissim FRANCEZ and Bartosz WIECKOWSKI (2014), A proof-theoretic semantics for contextual definiteness, in Enrico MORICONI and Laura TESCONI, editors, Second Pisa Colloquium in Logic, Language and Epistemology, ETS, Pisa, Italy.

Gottlob Frege (1884), Die Grundlagen der Arithmetik [The basic laws of arithmetics], Georg Olms, Hildesheim, Germany.

Gerhard GENTZEN (1969), Investigations into logical deduction, in M.E. SZABO, editor, The collected papers of Gerhard Gentzen, pp. 68-131, North-Holland, Amsterdam, Netherlands, English translation of the 1935 paper in German.

Michael GLANZBERG (2006), Context and unrestricted quantification, in Augustìne RAYO and Gabriel UzQUIANo, editors, Absolute Generality, Clarendon Press, Oxford, UK. 
Michael Moortgat (1997), Categorial type logics, in Johan VAN BENTHEM and Alice TER MEULEN, editors, Handbook of Logic and Language, pp. 93-178, North-Holland, Amsterdam, Netherlands.

Lawrence Moss (2010), Syllogistic logics with verbs, Journal of Logic and Computation, 20(4):947-967.

Francis Jeffrey Pelletier (2003), Context dependence and compositionality, Mind \& Language, 18(2):148-161.

Dag Prawitz (1965), Natural deduction: A proof-theoretical study, Almqvist and Wicksell, Stockholm, Sweden, soft cover edition by Dover, 2006.

Dag PRAWITZ (2006), Meaning approached via proofs, Synthese, 148:507-524. Arthur N. PRIOR (1960), The runabout inference-ticket, Analysis, 21:38-39.

Peter SCHROEDER-HEISTER (1984), A natural extension of natural deduction, Journal of Symbolic Logic, 49:1284-1300.

Jason STANLEY and Zoltán Gendler SzABó (2000), On quantifier domain restriction, Mind \& Language, 2-3:219-261.

Neil Tennant (1997), The taming of the true, Oxford University Press, Oxford, UK.

Jan VON PlAto (2000), A problem with normal form in natural deduction, Mathematical Logic Quarterly, 46:121-124.

Jan Von Plato (2001), Natural deduction with general elimination rules, Archive for Mathematical Logic, 40:541-567.

Dag WESTERSTÅHL (1985), Determiners and context sets, in Johan VAN BENTHEM and Alice TER MEULEN, editors, Generalized Quantifiers in Natural Language, Foris, Dordrecht, Netherlands.

This work is licensed under the Creative Commons Attribution 3.0 Unported License. http://creativecommons.org/licenses/by/3.๑/ 